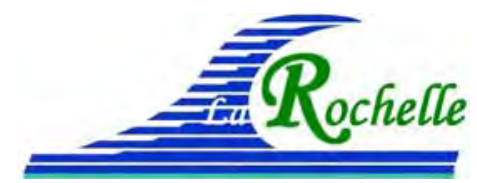

XVèmes Journées Nationales Génie Côtier - Génie Civil

La Rochelle, 29 au 31 mai 2018

DOI:10.5150/jngcgc.2018.036

(C) Editions Paralia CFL

disponible en ligne - http://www.paralia.fr - available online

\title{
Suivi vidéo de la dynamique hydro-sédimentaire côtière : exemples d'applications sur le littoral méditerranéen
}

\section{Yann BALOUIN ${ }^{1}$, Rémi BELON ${ }^{2}$, Clément BOUVIER ${ }^{1.3}$, Eric PALVADEAU ${ }^{1}$, Alexis STEPANIAN ${ }^{4}$, Julie MUGICA ${ }^{5}$}

1. BRGM, Université de Montpellier, 1039, rue de Pinville, 34000 Montpellier, France. y.balouin@brgm.fr ; e.palvadeau@brgm.fr ; c.bouvier@brgm.fr ;

2. BRGM, 5 rue Sainte-Anne, 97404 Saint-Denis, La Réunion, France. r.belon@brgm.fr

3. CNRS, UMR EPOC, Univ. Bordeaux, Allée Geoffroy Saint-Hilaire, CS 50023, 33615 Pessac Cedex, France. clement.bouvier@u-bordeaux.fr

4. BRGM, 117 avenue de Luminy - BP 168, 13276 Marseille Cedex 9, France.

a.stepanian@brgm.fr

5. BRGM, Immeuble Agostini, Zone Industrielle de Furiani, 20600 Bastia, France.

j.mugica@brgm.fr

\section{Résumé :}

Le suivi vidéo de la dynamique littorale s'est développé depuis les années 80 et de nombreux outils ont été mis en place pour analyser les signatures optiques des morphologies côtières et de l'hydrodynamique. L'intérêt majeur du suivi vidéo réside dans l'acquisition à haute fréquence et en continu, y compris lors des tempêtes, fournissant des données originales quantitatives inaccessibles à l'heure actuelle avec les moyens de suivi classiques dont on dispose. En méditerranée, des dispositifs à différentes résolutions ont été utilisés ces dernières années pour le suivi du littoral et la compréhension des mécanismes hydrosédimentaires. L'objectif de ce travail est de présenter, au travers d'exemples utilisant différents systèmes vidéo, les développements récents réalisés en termes de traitement et d'analyse pour le suivi du littoral :

- Suivi de l'évolution de la plage du Lido de Sète suite à la mise en place d'un atténuateur de houle, avec notamment, le suivi à haute fréquence de la dynamique des barres d'avant-côte ;

- Suivi de l'évolution de la plage du Barcarès suite à l'implantation de brise-lames, et notamment l'évolution contrastée de la plage à haute fréquence lors d'épisodes de tempêtes et de de forte tramontane ;

- La dynamique (dépôt/érosion) des banquettes à posidonies sur plusieurs plages de Corse, et son rôle sur l'érosion côtière.

Mots-clés : Suivi vidéo, Bathymétrie, Protection contre l'érosion, Banquettes à posidonies. 


\section{Thème 3 - Instrumentation, mesures, imagerie et télédétection}

\section{Introduction}

Le suivi vidéo de la dynamique littorale s'est développé depuis les années 80 (HOLMAN \& STANLEY, 2007), et de nombreux outils ont été mis en place pour analyser les signatures optiques des morphologies côtières et de l'hydrodynamique. L'intérêt majeur du suivi vidéo réside dans l'acquisition à haute fréquence et en continu, y compris lors des tempêtes, fournissant des données originales quantitatives inaccessibles à l'heure actuelle avec les moyens de suivi classiques dont on dispose (levés bathymétriques, mesures de houle, courants).

Différents systèmes sont actuellement à disposition : des appareils time-lapse bon marché à des systèmes éprouvés comme ARGUS, VM4GIS, CAM-ERA, ... . On peut ainsi avoir rapidement accès à des indicateurs qualitatifs de l'état du littoral, ou par des traitement plus complexes à des indicateurs quantitatifs tels que les vagues, les courants, la bathymétrie.

En Méditerranée, des dispositifs à différentes résolutions ont été utilisés ces dernières années pour le suivi du littoral et la compréhension des mécanismes hydrosédimentaires, en réponse notamment à des aménagements côtiers (atténuateur de houle, brise-lames) ou à la dynamique des banquettes de feuilles de posidonies déposées sur les plages.

\section{Méthodes}

Selon les objectifs de suivis, plusieurs techniques vidéo sont à disposition, plus ou moins couteuses, plus ou moins faciles à mettre en œuvre, et avec des résolutions plus ou moins fines. Le choix de la technique doit donc répondre à des critères tels que : la superficie de la zone à suivre, la durée du suivi, les contraintes d'installation, les besoins en termes de résolution qui sont très variables selon les applications et la dynamique des phénomènes observés.

Quel que soit le choix du matériel, la méthode consiste à installer sur le littoral des caméras à haute résolution, suffisamment en hauteur, et à une distance proche des morphologies à observer. Un levé de points de contrôle par GPS est effectué sur le site pour obtenir les informations indispensables au traitement. Des techniques de photogrammétrie sont par la suite utilisées pour rectifier et géo-référencer les images obtenues. On peut alors mettre en œuvre des traitements thématiques de ces images rectifiées. Ces algorithmes, développés sous Matlab ${ }^{\circledR}$, permettent par exemple la détection automatique du trait de côte (ARMAROLI et al., 2006), l'analyse des vagues et des courants de surface (HOLMAN \& STANLEY, 2007), la détection des barres sableuses sous-marines (BALOUIN \& GERVAIS, 2013 ; BOUVIER et al., 2017), dans certains cas la topographie émergée (BALOUIN et al., 2004), et la bathymétrie (HOLMAN et al., 2013), et enfin la reconnaissance d'objets permettant par exemple le comptage des usagers sur la plage (BALOUIN et al., 2014) ou la détection des banquettes à posidonies ou à sargasses. 


\section{XVèmes Journées Nationales Génie Côtier - Génie Civil \\ La Rochelle, 29 au 31 mai 2018}

\section{Applications sur le littoral méditerranéen}

L'objectif de ce travail est de présenter, au travers d'exemples utilisant différents systèmes vidéo, les développements récents réalisés en termes de traitement et d'analyse pour le suivi du littoral. Trois technologies ont été utilisées ces dernières années sur le littoral méditerranéen : le dispositif ARGUS à Sète, le dispositif VM4GIS au Barcarès et des systèmes low cost sur le littoral de Corse (figure 1).

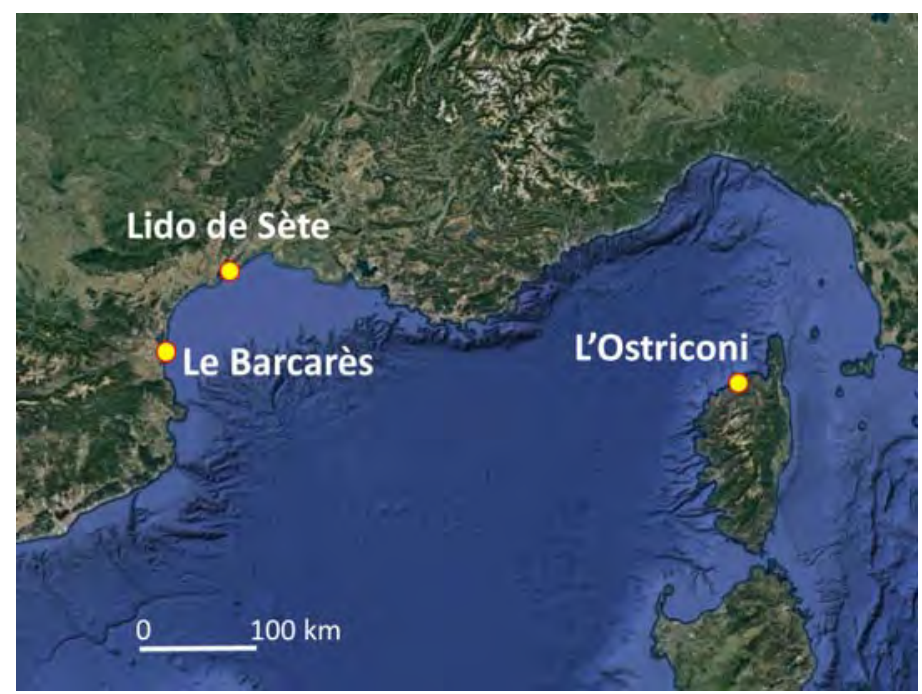

Figure 1. Localisation des sites d'étude.

\subsection{Suivi du Lido de Sète à Marseillan}

La plage du Lido de Sète a fait l'objet d'un important programme d'aménagement ces dernières années, avec le recul de la route littorale, la réhabilitation de la plage et du cordon dunaire, et l'expérimentation de techniques de lutte contre l'érosion côtière. Afin de suivre cette expérimentation, un dispositif ARGUS a été mis en place en 2011. Il est constitué de 2 mats de 20 m supportant 8 caméras et permettant le suivi en continu de 4 $\mathrm{km}$ de plage du triangle de Villeroy aux trois digues.

Ce dispositif permet le suivi de la position du trait de côte, des barres d'avant-côte, mais sa résolution permet également le suivi des forçages hydrodynamique et la reconstitution de la bathymétrie. Pour cette dernière, la technique cBathy (HOLMAN et al., 2013) s'appuie sur l'observation du champ de vagues de surface. Cet outil permet d'obtenir la profondeur d'eau en se basant sur la relation linéaire de dispersion :

$$
\sigma^{2}=g k \tanh (k h)
$$

Où $\sigma$ et $k$ sont la fréquence ( $2 \pi T$ avec T la période des vagues) et le nombre d'onde ( $2 \pi \lambda$ avec $\lambda$ la longueur d'onde des vagues) respectivement. $g$ est la gravité et $h$ la profondeur. L'algorithme consiste à estimer la vitesse de propagation des vagues $(\sigma / \mathrm{k})$ qui permettra d'obtenir la profondeur $h$. 


\section{Thème 3 - Instrumentation, mesures, imagerie et télédétection}

L'application de cet outil sur le littoral de Sète permet d'obtenir des résultats très intéressants qui sont toujours en cours de validation (levé bathymétrique programmé début 2018 pour une estimation fine des erreurs). La figure 2 présente ainsi le résultat pour la journée du 15 Janvier 2018. Les résultats mettent en évidence une influence forte du vent, de la période de la houle qui est en cours d'analyse pour déterminer les conditions les plus favorables à la reconstruction bathymétrique.

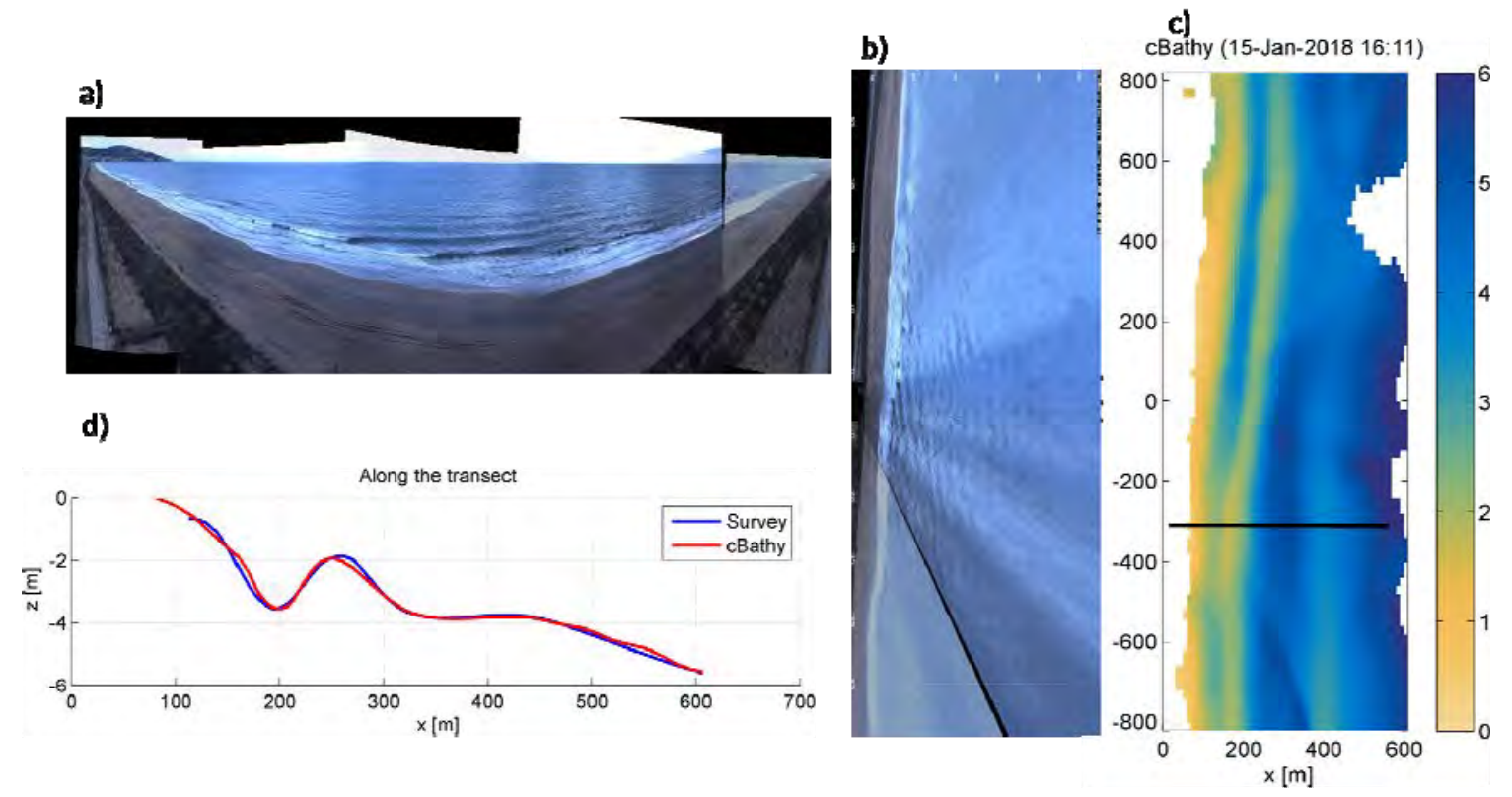

Figure 2. Résultat de la reconstruction bathymétrique du 15 janvier 2018 :

a) panorama des images brutes; b) image rectifiée, c) résultats de cBathy, et

d) comparaison d'un profil bathymétrique avec un profil cBathy.

\subsection{Suivi de la plage du Barcarès}

Sur la plage du Barcarès, dans les Pyrénées-Orientales, un dispositif VM4GIS (RIHOUEY et al., 2009) a été mis en place en 2014 suite à l'implantation de 3 briselames. L'objectif de ce suivi réside dans la compréhension des modifications de la dynamique littorale par ces ouvrages, et dans l'évaluation de leur efficacité.

Les figures 3 et 4 présentent le suivi temporel de la position du trait de côte en fonction des conditions météo-marines (épisodes de tramontane et tempêtes marines), ainsi que les phases de rechargement de plage qui sont réalisées annuellement depuis la mise en place des brise-lames. Les analyses ont notamment permis de mieux comprendre le rôle des forçages météo-marins sur le développement et la dynamique des tombolos derrière les brise-lames. Les tempêtes marines tendent à linéariser le trait de côte, alors que les épisodes de tramontane (vent quasi-parallèle au trait de côte) ont tendance à développer les tombolos. Ces variations semblent s'effectuer à volume sableux constant, bien que le volume global (levés bathymétriques non présentés ici) est en augmentation du fait de rechargements réguliers (cf. figure 3). 


\section{XVèmes Journées Nationales Génie Côtier - Génie Civil \\ La Rochelle, 29 au 31 mai 2018}

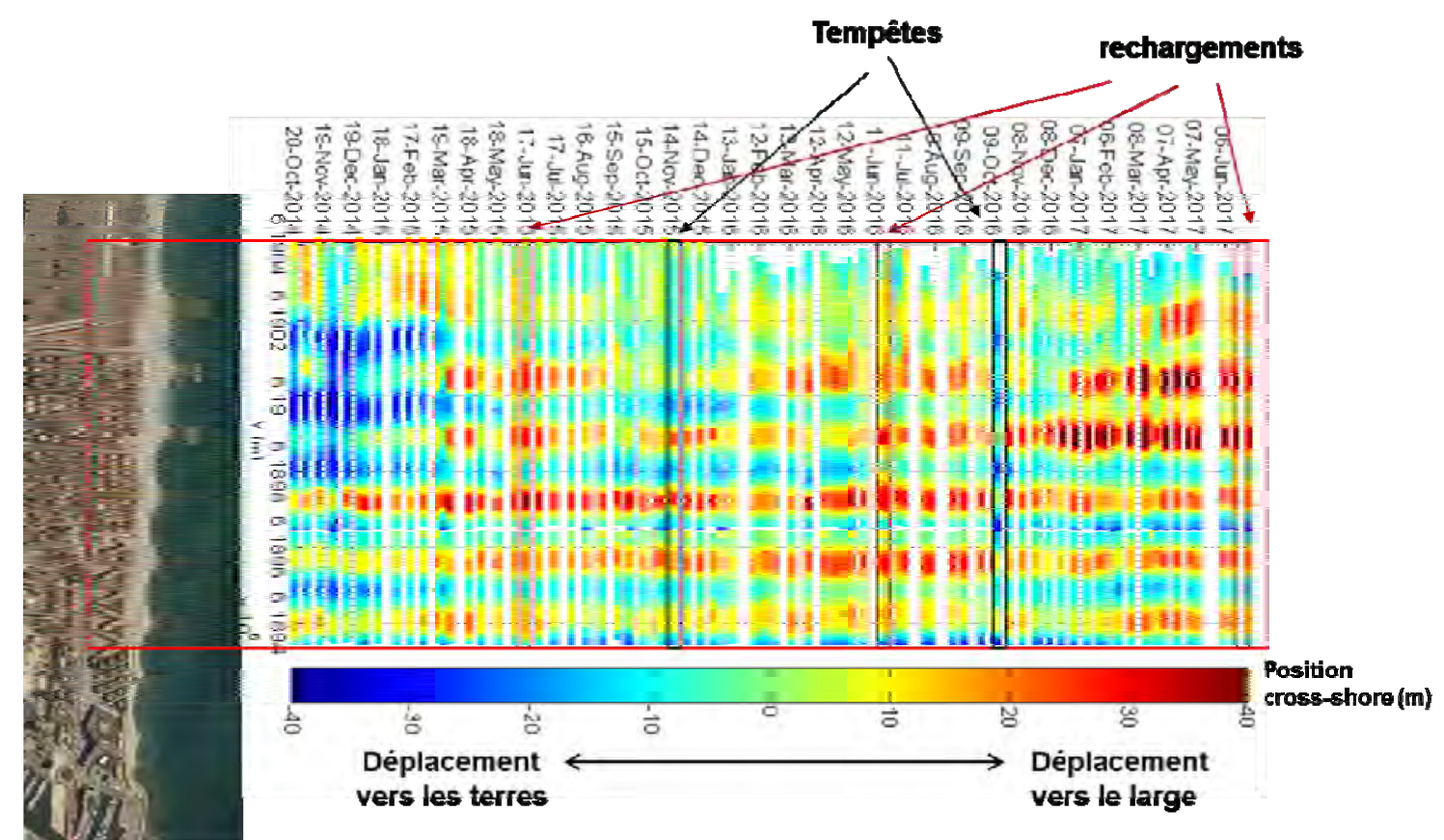

Figure 3. Evolution temporelle et spatiale de la position du trait de côte sur la plage du Barcarès.

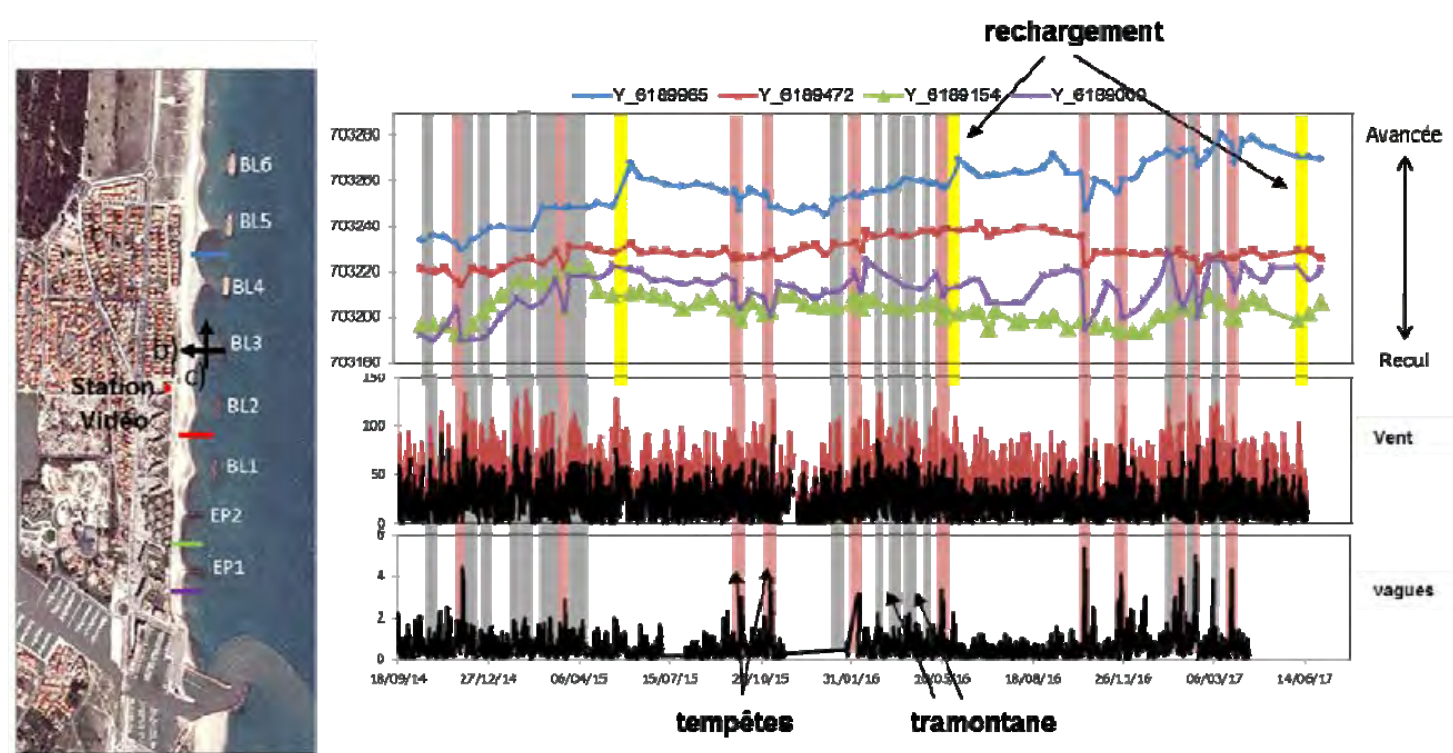

Figure 4. Evolution temporelle de la position du trait de côte le long de quelques profils transverses (localisés sur la photographie à gauche), en fonction des conditions de vent, de houle et des rechargements en sable sur la plage du Barcarès.

\subsection{Suivi de la plage de l'Ostriconi en Corse}

Sur le littoral de Corse, des appareils photo time-lapse (CSolarcam) ont été disposés sur plusieurs plages pour analyser le rôle des banquettes à posidonies sur l'évolution du trait 


\section{Thème 3 - Instrumentation, mesures, imagerie et télédétection}

de côte. L'objectif était à la fois de mieux comprendre la dynamique des banquettes (érosion/dépôt), mais également d'évaluer l'effet protecteur de ces banquettes pendant les tempêtes. Des outils de traitement similaires à ceux des systèmes vidéo ont été développés, et si ces appareils ont une résolution nettement moins bonne que les systèmes vidéo précédents, ils permettent tout de même d'obtenir des informations quantitatives intéressantes sur les petites plages de poche de Corse.

Les figures 5 et 6 présentent un exemple de traitements réalisés sur la plage de l'Ostriconi, où des banquettes de quelques décimètres d'épaisseur sont présentes. Les résultats ont notamment permis de caractériser l'influence de la direction de la houle sur les dépôts/érosion des banquettes, ainsi que les phénomènes de basculement des plages lors de vagues obliques. Lors du premier événement, du 1 au 3 avril 2017, le pic de vague entraine un recul du trait de côte et de la banquette sur les deux côtés de la baie. Toutefois, les vagues de nord sur le tombant du coup de mer permettent la reconstruction du profil (avancée d'environ $15 \mathrm{~m}$ ) au sud alors que le profil nord reste déficitaire. Lors du second événement une semaine plus tard, le phénomène inverse est observé avec un transfert sédimentaire du sud vers le nord sous l'influence de la houle de sud-ouest. Ce type de basculement des plages de poches, largement décrit dans la littérature est toutefois observé pour des conditions d'agitation modérées, et se produit très rapidement. Il aurait difficilement pu être caractérisé par des suivis topobathymétriques classiques. Les résultats permettent également d'attester du rôle protecteur de ces banquettes lors des tempêtes, qui reste toutefois à investiguer plus en détail.

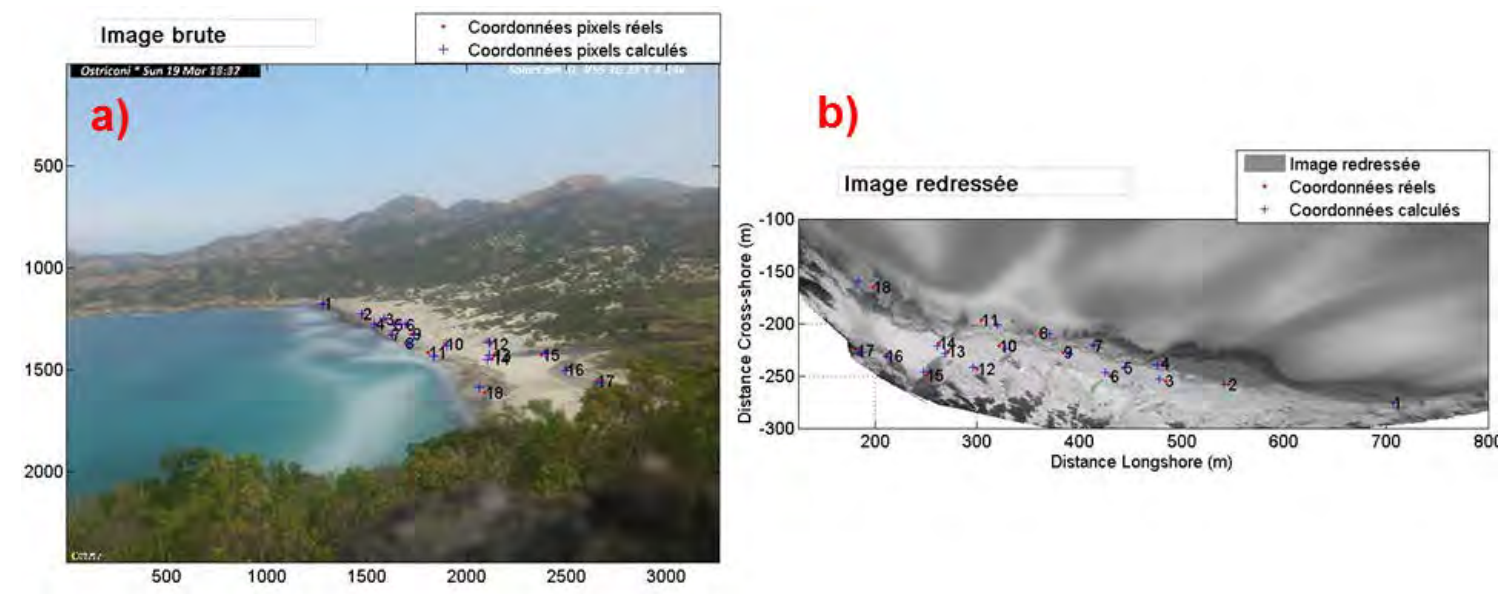

Figure 5. Traitement réalisés sur les images low cost sur la plage de l'Ostriconi :

a) image brute moyennée sur 10 min, b) image redressée. 


\section{XVèmes Journées Nationales Génie Côtier - Génie Civil La Rochelle, 29 au 31 mai 2018}
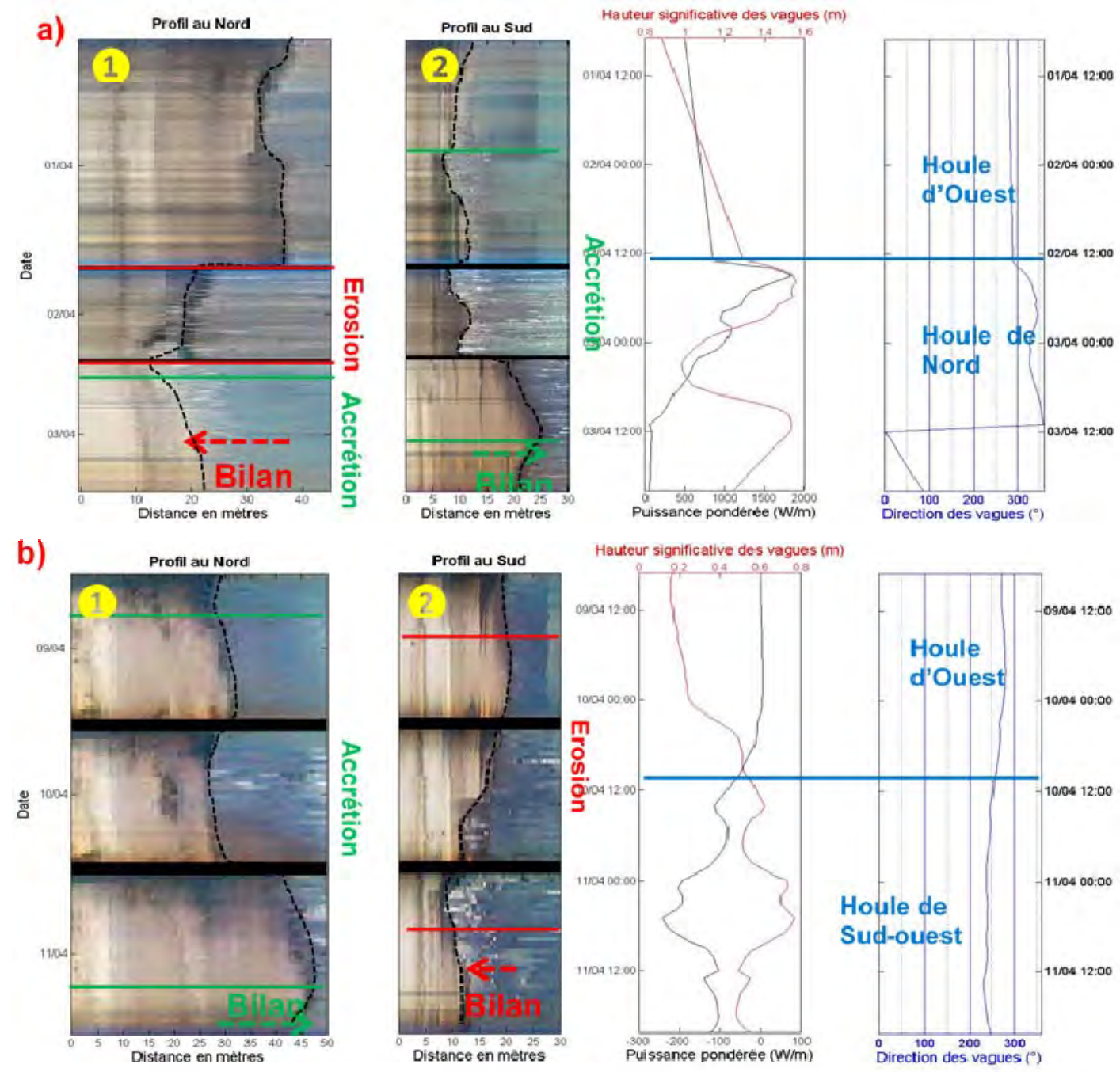

Figure 6. Analyse temporelle (time-stack) de profils transverses situés au nord et au sud de la baie : a) coup de mer du 01 au 03-04-2017, b) période du 09 au 11/04/2017 mettant en évidence la mobilité du trait de côte (basculement nord-sud) et de la banquette au cours d'une tempête.

\section{Conclusion}

Les systèmes d'observation vidéo du littoral sont en plein développement ces dernières années. Les résultats présentés ici montrent le potentiel de ces outils pour obtenir des données quantitatives en continu, y compris pendant les épisodes de tempêtes pendant lesquels les mesures in-situ restent difficiles et risquées (pour le matériel et les hommes). Les développements récents en termes d'inversion bathymétrique offrent un potentiel important pour l'assimilation des données dans les modèles numériques et la mise en œuvre de systèmes d'alerte s'appuyant sur des données actualisées par vidéo. 
Thème 3 - Instrumentation, mesures, imagerie et télédétection

\section{Remerciements}

Le système vidéo du Lido de Sète est financé par Thau Agglopôle et le BRGM. Le suivi du Barcarès est réalisé dans le cadre de l'Observatoire de la côte sableuse catalane (http://obscat.fr), et le suivi réalisé sur le littoral de Corse est financé par le BRGM et la DDTM-2B.

\section{Références bibliographiques}

ARMAROLI C., BALOUIN Y. , CIAVOLA P., GATTI M. (2006). An integrated study of shoreline variability using GIS and ARGUS techniques. Journal of Coastal Research, SI39, ICS2004.

BALOUIN Y., GERVAIS M. (2013). Morphological feedback between nearshore bars and shoreline dynamics during storm events - video observations at Sète beach, France. Proceedings of $7^{\text {th }}$ Coastal Dynamics Conférence, Arcachon, 24-28 June 2013, pp 151-160.

BALOUIN Y., MORRIS B.D., DAVIDSON M.A., HOWA H. (2004). Morphology evolution of an ebb-tidal delta following a storm perturbation: assessments from remote sensed video data and direct surveys. Journal of Coastal Research, Vol. 20(2), pp 415-423. https://doi.org/10.2112/1551-5036(2004)020[0415:MEOAED]2.0.CO;2

BALOUIN Y., REY-VALETTE H., PICAND P.-A. (2014). Automatic assessment and analysis of beach attendance using video images at the Lido of Sète beach, France. Ocean and Coastal Management, Vol. 102(A), pp 114-122. https://doi.org/10.1016/j.ocecoaman.2014.09.006

BOUVIER C., BALOUIN Y., CASTELLE B. (2017). Video monitoring of sandbarshoreline response to an offshore submerged structure at a microtidal beach. Geomorphology, Vol. 295, pp 297-305. https://doi.org/10.1016/j.geomorph.2017.07.017

HOLMAN R.A., STANLEY J. (2007). The history and technical capabilities of Argus. Coastal Engineering, Vol. 54(6-7), pp 477-491. https://doi.org/10.1016/j.coastaleng.2007.01.003

HOLMAN R., PLANT N., HOLLAND T. (2013). cBathy: A robust algorithm for estimating nearshore bathymetry. Journal of Geophysical Research, Vol. 118(5), pp 2595-2609. https://doi.org/10.1002/jgrc.20199

RIHOUEY D., DUGOR J., DAILLOUX D., MORICHON D. (2009). Application of remote sensing video systems to engineering works monitoring. Journal of Coastal Research, Vol. SI 56, pp 1582-1586. 